\title{
Classifying Imaginary Hand Movement through Electroencephalograph Signal for Neuro-rehabilitation
}

\author{
Osmalina RAHMA ${ }^{1, *}$, Rimuljo HENDRADI ${ }^{2}$ and Fadli AMA ${ }^{1}$ \\ ${ }^{1}$ Biomedical Engineering, Department of Physics, Faculty of Science and Technology, \\ Universitas Airlangga, Indonesia \\ ${ }^{2}$ Information System, Department of Mathematics, Faculty of Science and Technology, \\ Universitas Airlangga, Indonesia
}

("Corresponding author's e-mail: osmalina.n.rahma@fst.unair.ac.id)

Received: 9 October 2017, Revised: 15 March 2018, Accepted: 30 April 2018

\begin{abstract}
Brain-Computer-Interface (BCI) has been widely used in the field of neuro-rehabilitation such as automatic controls based on brain commands to upper and lower extremity prosthesis devices in patients with paralysis. In a post-stroke period, approximately $50 \%$ of stroke survivors have unilateral motor deficits leading to a sustained decline in chronic upper extremity function. Stroke affects patients in their productive and elderly age which is potentially creating new problems in national health development. BCI could be used to aid post-stroke patient recovery, so the motion detection and classification are essential for optimizing the BCI device control. Therefore, this study aims to distinguish several hand functions such as grasping, pinching, and hand lifting from releasing movement by the standard actions performed during post-stroke rehabilitation based on brain signals of a healthy subject obtained from an electroencephalogram (EEG) with a 5 channels electrode. In this study, the EEG signals were decomposed using a Discrete Wavelet Transform (DWT) then filtered by a bandpass filter to generate Mu and Beta waves, which were correlated with imaginary movement. Then, the $\mathrm{Mu}$ and Beta waves were calculated using a Common Spatial Pattern (CSP) algorithm as the inputs for Extreme Learning Machine (ELM) to distinguish 2 types of imaginary hand movements (grasping $v$. releasing, pinching $v$. releasing, hand lifting $v$. releasing). The results of these classifications shown that ELM and CSP were useful features in distinguishing 2 types of motion with software/system accuracy average above $95 \%$. Therefore, this could be useful for optimizing BCI devices in neuro-rehabilitation, moreover by combining it with a Functional Electrical Stimulator (FES) as a self-therapy for post-stroke patients.
\end{abstract}

Keywords: Brain-Computer-Interface (BCI), neuro-rehabilitation, electroencephalogram (EEG)

\section{Introduction}

Stroke is a brain functional disease in the form of impaired neural function locally or globally and appears suddenly, progressively, and quickly [1]. Strokes may cause partial paralysis of the body, and after a healing period or post-stroke period, about $50 \%$ of stroke survivors have unilateral motor deficits that lead to decreased chronic upper extremity function [2]. This motor deficit affects hand or arm function of the post-stroke patients' body in everyday life even several years after the stroke. Both active and passive motoric rehabilitation (with the aid of a tool or therapist) is necessary to restore hand/arm function as they were before.

The Brain-Computer-Interface (BCI) or Brain-Machine-Interface (BMI) uses brain signals to run a device in the absence of motoric system participation [3]. BCI or BMI has been extensively developed in neuro-rehabilitation to help post-stroke patients restore upper or lower extremity function by combining it with Functional Electrical Stimulation (FES), robots, and orthotic. Daly combined BCI and FES for post- 
http://wjst.wu.ac.th

stroke patient therapy based on the imaginary movement of finger extension [4]. Ang et al. reported combining BCI and Haptic Knop robot (HK) for hand rehabilitation of stroke patients based on the imaginary movement of grasp [5]. Moreover, Buch who did a BCI research in stroke patients based on the imaginary movement of grasp combined with orthoses [6].

BCIs could be used for post-stroke patient recovery as a tool for aiding patient in the rehabilitation, so motion detection and classification are essential for optimizing the BCI device control. However, the conventional problem of obtaining data from stroke survivors is that they do not have the physical condition to complete all the process. As a result, most BCIs studies are designed using a subjectdependent approach using healthy subjects [7]. Besides, the fewer electrodes would be more comfortable than 32 or 64 electrodes. Therefore, this study aims to distinguish several hand functions such as grasping, pinching, and hand lifting by standard movements performed during post-stroke rehabilitation based on brain signals of a healthy subject obtained from a 5-channels electrode EEG.

BCI followed by machine learning has been proven effective with minimal training required and compensates for the high variability among subjects [8]. In this study, the information obtained from the EEG after signals processing, such as Wavelet transform and bandpass filter, were used as inputs for neural networks to distinguish 2 types of imaginary hand movements (grasping $v$. releasing, pinching $v$. releasing, hand lifting $v$. releasing). The neural network used in this study was a type of feed-forward neural network with an Extreme Learning Machine (ELM) algorithm because it has a faster training time and higher accuracy compared to other algorithms such as Back Propagation and Support Vector Machines (SVM) in the signal classification [9].

\section{Materials and methods}

\section{Experiment protocol}

BCIs are a subject-dependent system which is subject-specific and need a calibration session for any new user. Thus, BCIs are only suitable for just one particular subject [10]. In this study, a healthy subject participated. The subject was in a conscious condition (eyes open) with both hands resting in their lap and was asked to imagine several types of hand movement, such as grasping, pinching, hand lifting, and hand releasing or relaxing in the right hand. Each task was performed 42 times with the same subject for $10 \mathrm{~s}$ approximately. Every consecutive task was separated with $10 \mathrm{~s}$ rest, while a different task was separated with 1 min rest $[11,12]$. This pause was meant to avoid fatigue due to the constant concentration. The order of the imaginary movement began with imagining the release movement or relaxing, then grasping, pinching and ended with hand lifting.

\section{Signal acquisition}

BCI is a system which does not depend on peripheral nerves and muscles. This definition limits the range of signals that control BCI is only from brain signals, excluding signals that obtained from muscles or peripheral nerves [13]. The signals were generated from electrodes that were placed on several points of the scalp which represent brain regions, such as frontal, temporal, central, parietal, and occipital. The data were obtained using an EEG EMOTIV Insight machine with 5 channel and $128 \mathrm{~Hz}$ sampling frequency (Figure 1). The electrode was positioned according to EMOTIV device which has 5 EEG sensors and 2 reference sensors as shown in Figure 2. These positions represent activity in the frontal cortex (AF3-AF4), the parietal-temporal cortex (T7-T8), and the parietal-occipital cortex (Pz).

\section{Signal processing}

The signals were processed as shown in the flow diagram (Figure 3). EEG recordings which were obtained with the EEG EMOTIV Insight machine were stored in the European data format (.edf) then converted to ASCII (American Standard Code for Information Interchange) data [14]. Thus, each recording was changed into a 1280-by-5 matrix (a 10-second-period of recordings-by-the number of channels). The ASCII data was filtered into 2 sub-waves based on the frequency range: $\mathrm{Mu}(7-15 \mathrm{~Hz})$ and beta $(15-25 \mathrm{~Hz})$ using the Discrete Wavelet Transform (DWT) at a 3 level decomposition with Daubechies $4(\mathrm{db} 4)$ as the mother Wavelet. The decomposition process is shown in Figure 4. 

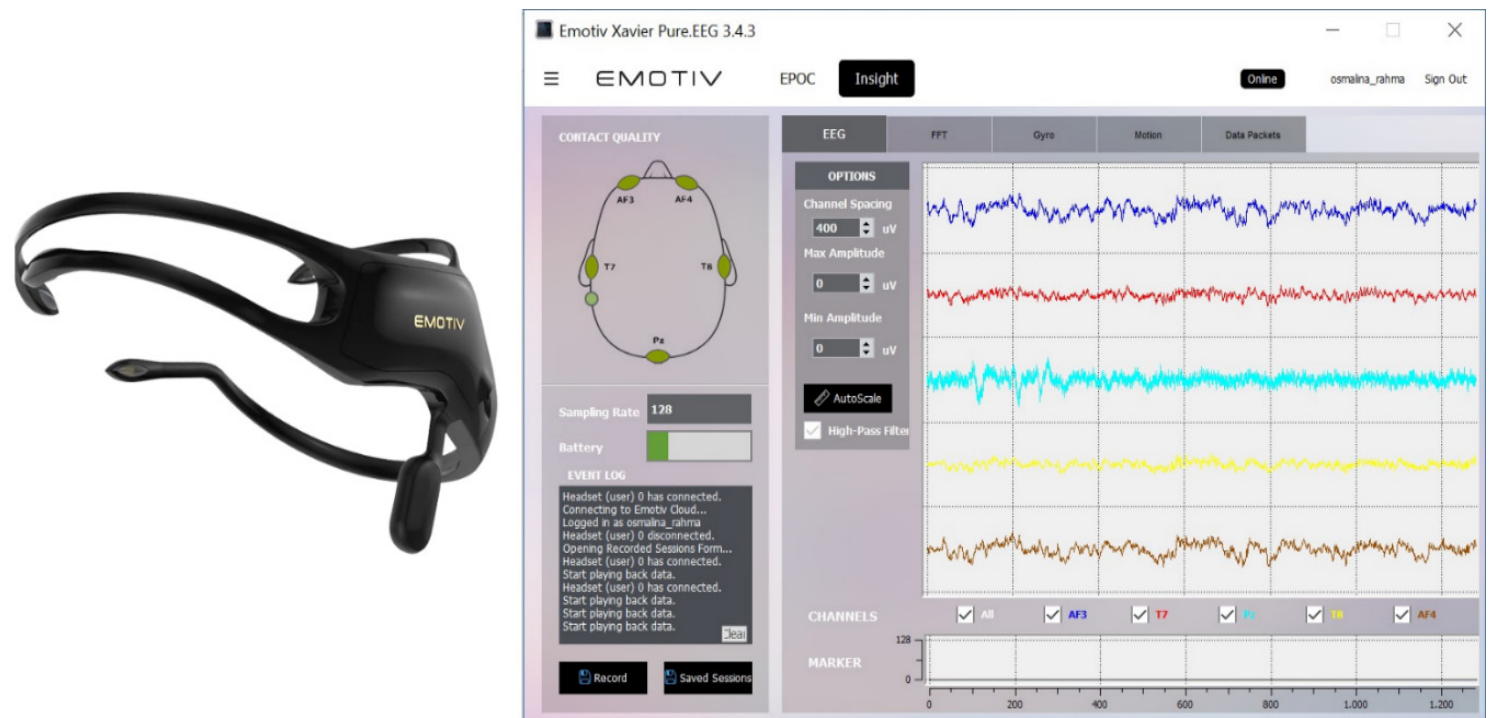

Figure 1 EEG signal recording.
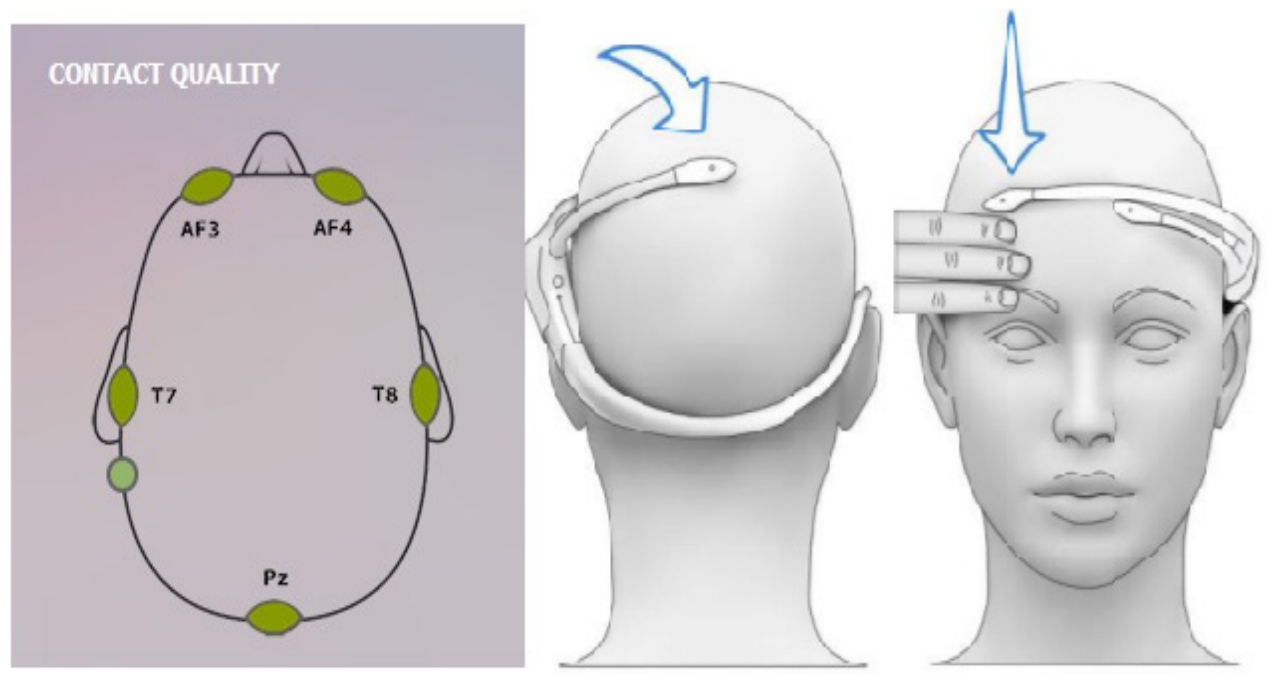

Figure 2 EMOTIV Insight electrode placement [20].

Based on Figure 4, the coefficients of D3 and D4 were consistent with the Mu and Beta frequency ranges. These coefficients were then filtered with the Equiripple Bandpass filter to make sure that these coefficients were in the correct frequency range for $\mathrm{Mu}(7-15 \mathrm{~Hz})$ and Beta $(15-25 \mathrm{~Hz})$. Afterward, the average power spectral density (PSD) values were calculated. The average PSD was obtained by summing the squared values of the energy in each segment to obtain a matrix with dimensions $1 \times 10$ ( 5 channels of Mu wave and 5 channels Beta wave) for each recording. 


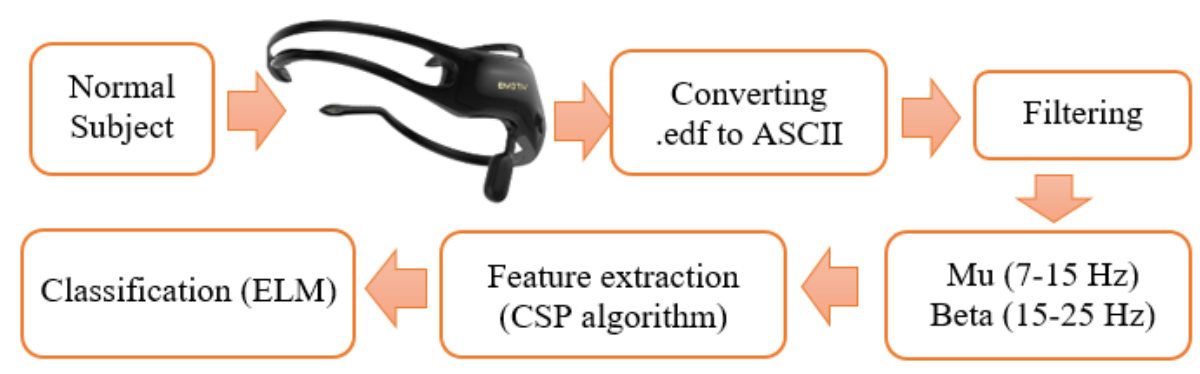

Figure 3 The Flow diagram of classifying imaginary hand movement.

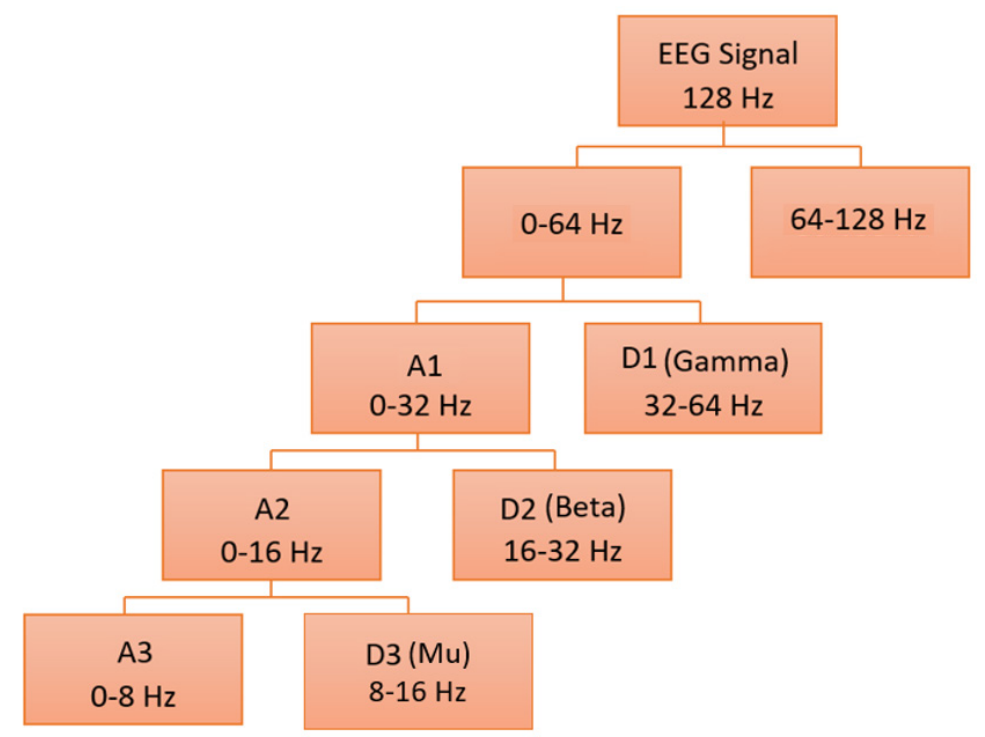

Figure 4 The Signal decomposition using DWT level 3.

\section{Feature extraction}

The next process was to calculate the CSP values that were used as features for the ELM neural network input (Figure 3). The CSP algorithm is a signal processing method that can be used to increase discriminatory classes and is an excellent method for BCI signal classification [13]. This algorithm calculates the variance of each class then maximizes the variance of one class, while at the same time minimizing the variance of the other classes. Thus, the distinction between classes is more apparent. This study used a modification of the CSP algorithm by first filtering the signal into 2 frequency bands, Mu and Beta wave then calculating its variance based on the CSP algorithm. The CSP was obtained from the script from Ethridge and Weaver [15]. We conducted a 30-by-10 matrix for 30 data of training and a 12by-10 matrix for 12 data testing.

\section{Classification}

The classification process (Figure 3) began with the normalization stage of feature values which used the "Mapminmax" function with syntax $[\mathrm{y}, \mathrm{PS}]=\operatorname{mapminmax}(\mathrm{x},-1,1)$, where $x$ and $y$ were the input data and the result of data normalization, respectively. The normalization results were the CSP value of $\mathrm{Mu}$ and Beta in the range of minus one and one [-1,1]. The training process was performed based on the normalization result as the feature values from each type of hand movement (grasping, pinching, 
http://wjst.wu.ac.th

hand lifting and relaxing or releasing movement) to obtain the input weights and the output weights used for the testing phase. These features were classified using the Extreme Learning Machine (ELM), which is a type of feedforward neural network with a single hidden layer which abandons the traditional algorithm that iteratively adapts all network parameters, yet determines the weights between hidden neurons and the output of neurons from a single hidden layer analytically. ELM uses Moore Penrose Pseudo Inverse theory to minimize the training error, and it has been shown to have higher accuracy compared to other types of ANN, such as Back Propagation and Support Vector Machine (SVM) [16]. Besides that, ELM parameters were selected at random so that it has fast learning and is able to produce high accuracy [17].

The optimal features were determined through $\mathrm{K}$-fold cross-validation with $\mathrm{K}=4$ (4-fold cross validation) so that the training and testing stage was done 4 times (fold) with different training and testing data than that used in the ELM. The average classification accuracy was calculated based on 5 different values of the hidden neural as the evaluations for each task pair of imaginary hand movements (grasping $v$. releasing, pinching $v$. releasing, hand lifting $v$. releasing). The ELM training was conducted with 60 data points, while the ELM testing was performed with 24 data which consisted of the same amount from each task, in both of training and testing.

\section{Results and discussion}

After converting into ASCII data, EEG recordings were then filtered into 2 sub waves based on the frequency range: $\mathrm{Mu}(7-15 \mathrm{~Hz})$ and beta $(15-25 \mathrm{~Hz})$ using the Discrete Wavelet Transform (DWT) decomposition technique with 4 decompositions and a mother Wavelet type Daubechies 4 (db4). The Mu and Beta waves were the frequencies that were correlated with left or right-hand motor imagery [11]. The coefficients of the decomposition products corresponding to the $\mathrm{Mu}$ and Beta frequency ranges were decompositions 2 and 3 (D2 and D3). Sub-bands were filtered with an Equiriple Bandpass filter in Mu wave in a frequency range 7 to $15 \mathrm{~Hz}$ (Figure 5a), and Beta at frequency range 15 to $25 \mathrm{~Hz}$ (Figure 5b) then calculated its average power spectral density (PSD).

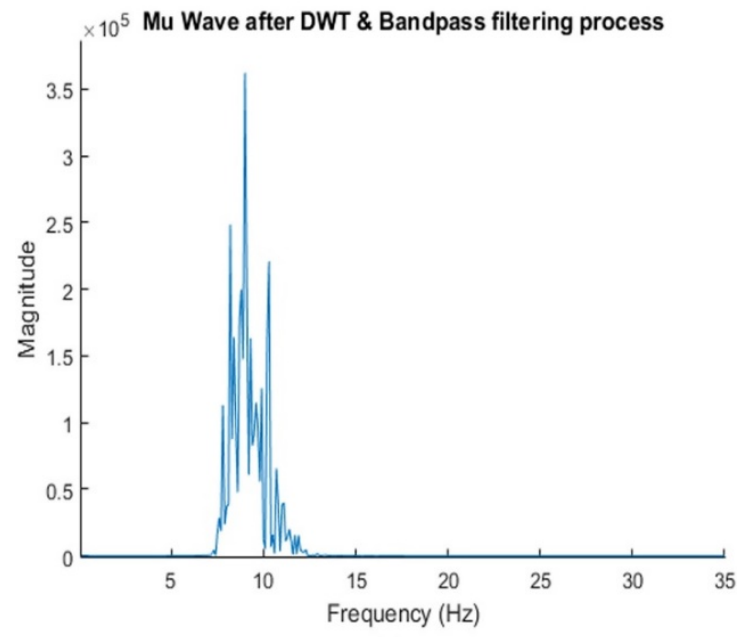

(a)

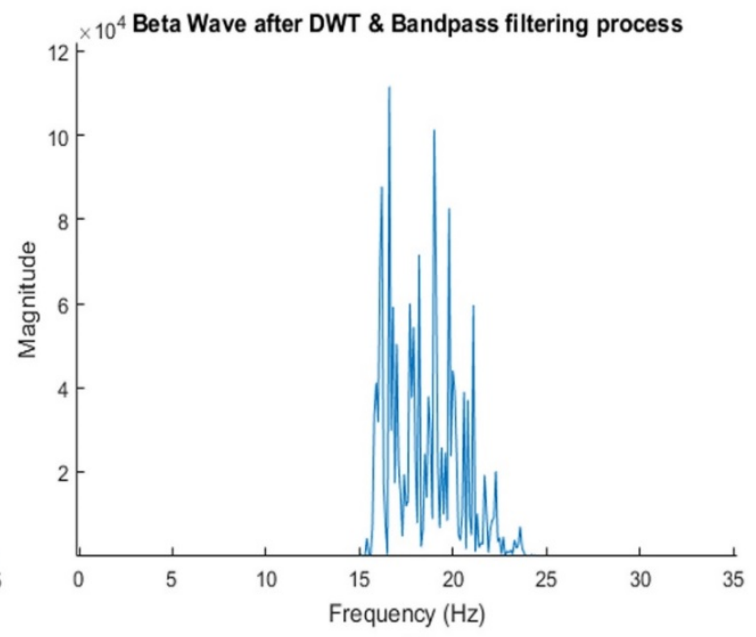

(b)

Figure 5 (a) Mu and (b) beta wave after DWT and band pass filtering process. 


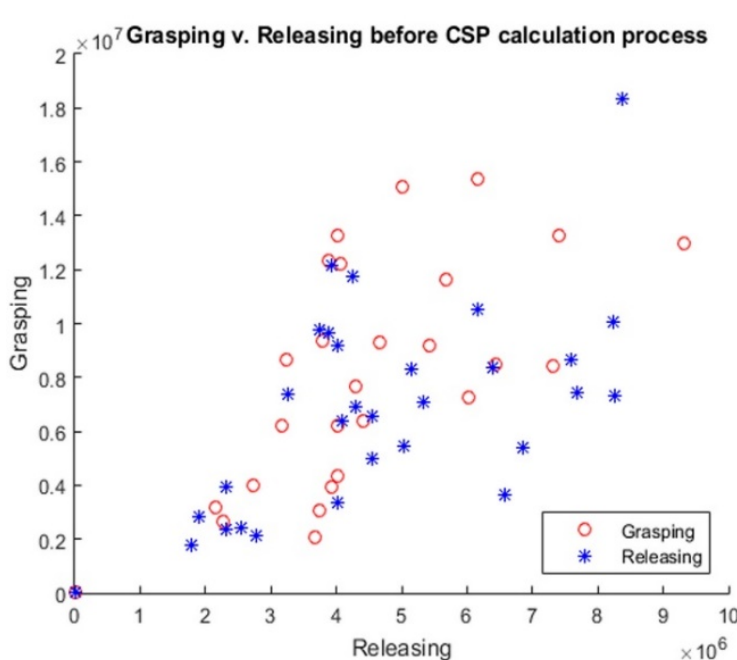

(a)

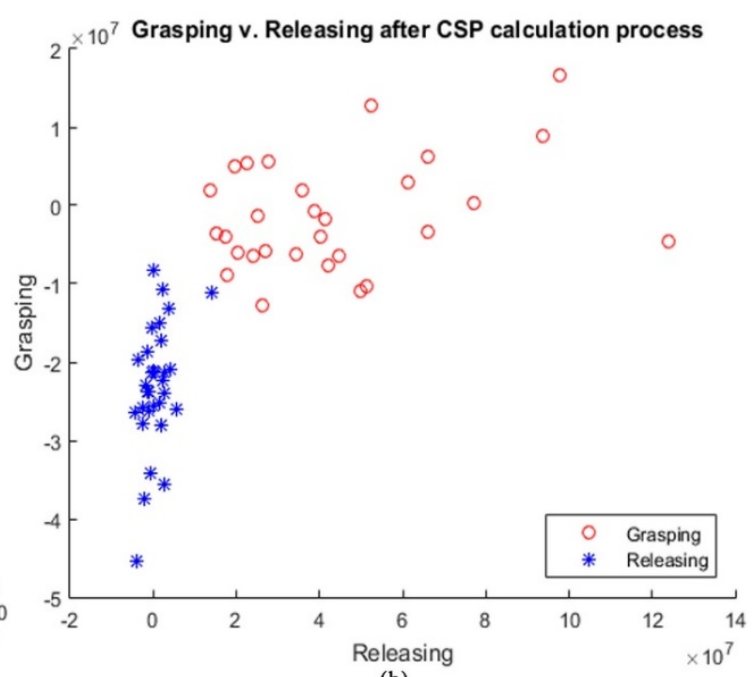

(b)

Figure 6 Distribution of data of grasping $v$. releasing (a) before and (b) after CSP calculation process.

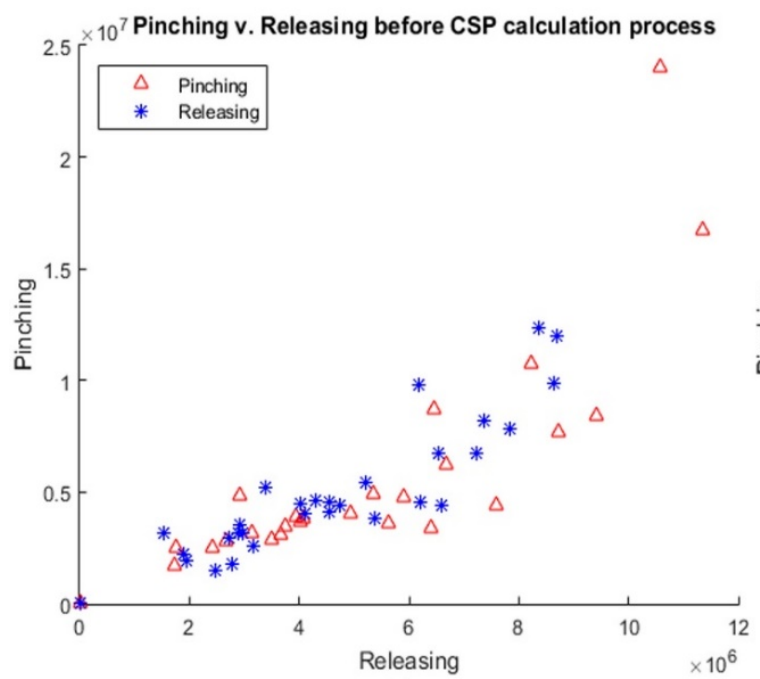

(a)

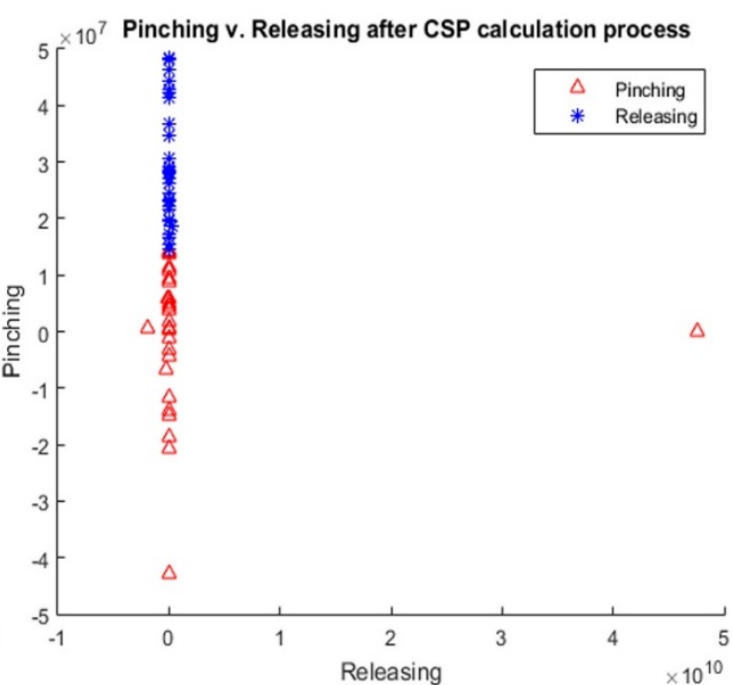

(b)

Figure 7 Distribution of data of pinching $v$. releasing (a) before and (b) after CSP calculation process. 


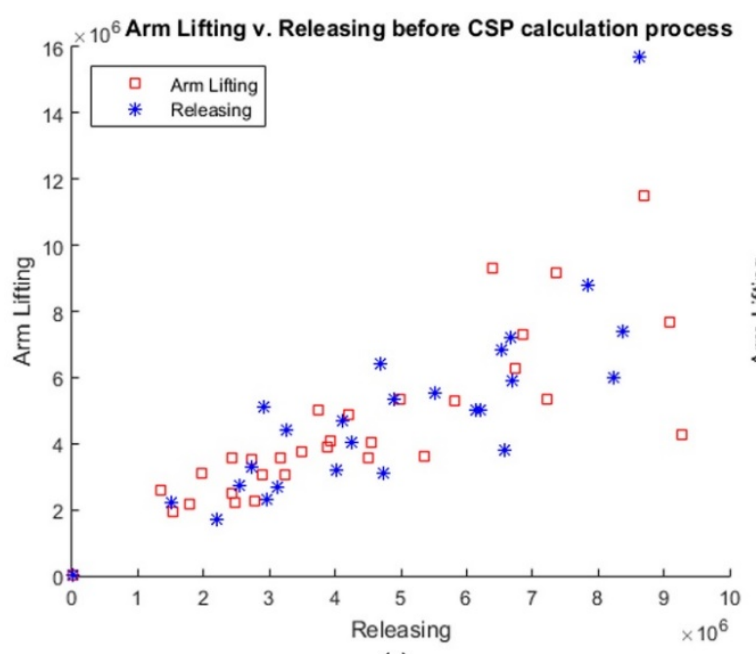

(a)

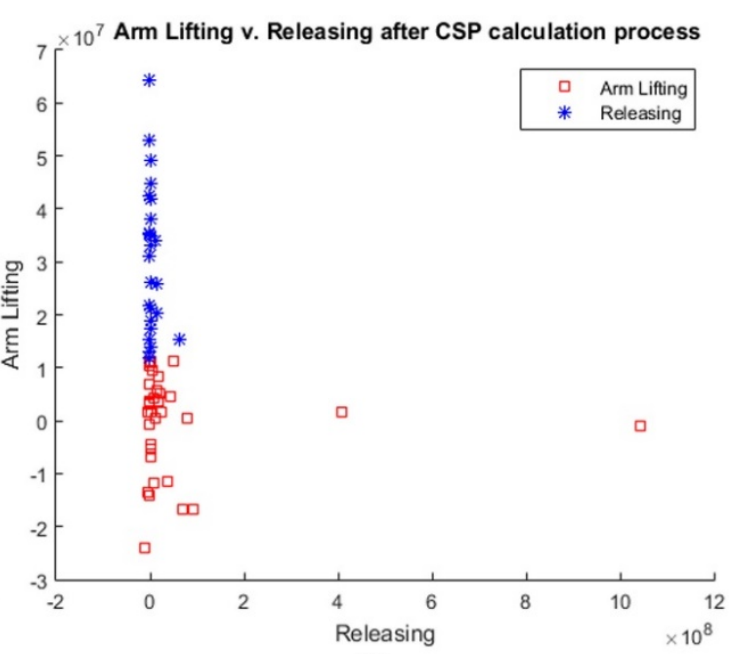

(b)

Figure 8 Distribution of data of hand lifting $v$. releasing (a) before and (b) after CSP calculation process.

The average PSD of $\mathrm{Mu}$ and Beta waves were calculated using a CSP algorithm to extract the features of the EEG signal as an input for the ELM. The CSP has been widely used in BCI since it can efficiently obtain information from 2 classes of imaginary movement. This algorithm maximizes the variance of one class and minimizes the other class simultaneously. Figure 6 indicates the distribution of data before and after CSP algorithm was implemented in 2 imaginary types of movement, grasping and releasing. Before calculating CSP values, grasping movement was difficult to separate from releasing movement. The same treatment was also applied in Figures 7 and $\mathbf{8}$ which show the distribution data of pinching versus releasing movement and the distribution data of hand lifting versus releasing movement, respectively. These figures indicate that CSP was proven in helping to separate 2 types of hand movement well in all classes (grasping $v$. releasing, pinching $v$. releasing, hand lifting $v$. releasing).

The following step after performing feature extractions by calculating the CSP values was the classification stage using ELM to distinguish 2 types of hand movement. The classification began with normalization, which changed the CSP value of $\mathrm{Mu}$ and Beta wave to within the range of -1 and 1 . Furthermore, the input weights and the output weights as a result of the training stage were carried out with 60 data consisting of 30 grasping/pinching/hand lifting data and 30 releasing movements data. The testing performed using 24 data comprised of 12 grasping/pinching/hand lifting data and 12 data of releasing movement based on the input and output weights from the training phase. The training and testing data were done using a 4-fold cross-validation method, so that, the training and testing phase were performed 4 times (fold) with different training data and testing data. System performance values of accuracy are shown in Tables $\mathbf{1} \mathbf{- 3}$, respectively. 
http://wjst.wu.ac.th

Table 1 shows the accuracy in classifying the grasping and releasing movement, while Table 2 represents the accuracy in distinguishing pinching and releasing movement and Table 3 details the accuracy in classifying of hand lifting and releasing hand movement. Based on all 3 tables, the minimum number of hidden neurons for 30 training data and 12 testing data was 50 because it had the highest training and testing accuracy value. The classification accuracies were $96 \%$ for classification of grasping $v$. releasing, $95 \%$ for classification of pinching $v$. releasing and $96 \%$ for classification of lifting hand $v$. releasing. The overall average performance accuracy was above $95 \%$ for the 2-class imaginary hand movement.

Table 1 Accuracy of the classification of grasping $v$. releasing.

\begin{tabular}{|c|c|c|c|c|c|c|c|c|c|c|}
\hline \multicolumn{11}{|c|}{30 Data of Training and 12 Data of Testing } \\
\hline \multirow{3}{*}{$\begin{array}{l}\text { Hidden } \\
\text { neuron }\end{array}$} & \multicolumn{10}{|c|}{ Accuracy } \\
\hline & \multicolumn{2}{|c|}{ Round 1} & \multicolumn{2}{|c|}{ Round 2} & \multicolumn{2}{|c|}{ Round 3} & \multicolumn{2}{|c|}{ Round 4} & \multicolumn{2}{|c|}{ Average } \\
\hline & $\begin{array}{c}\text { Train } \\
(\%)\end{array}$ & $\begin{array}{l}\text { Test } \\
(\%)\end{array}$ & $\begin{array}{c}\text { Train } \\
(\%)\end{array}$ & $\begin{array}{l}\text { Test } \\
(\%)\end{array}$ & $\begin{array}{c}\text { Train } \\
(\%)\end{array}$ & $\begin{array}{l}\text { Test } \\
(\%)\end{array}$ & $\begin{array}{c}\text { Train } \\
(\%)\end{array}$ & $\begin{array}{l}\text { Test } \\
(\%)\end{array}$ & $\begin{array}{c}\text { Train } \\
(\%)\end{array}$ & $\begin{array}{l}\text { Test } \\
(\%)\end{array}$ \\
\hline 1 & 50.00 & 50.00 & 50.00 & 50.00 & 50.00 & 50.00 & 50.00 & 50.00 & 50.00 & 50.00 \\
\hline 5 & 91.67 & 62.50 & 90.00 & 87.50 & 98.30 & 95.83 & 93.33 & 70.83 & 93.33 & 79.16 \\
\hline 10 & 95.00 & 66.67 & 93.33 & 87.50 & 100.00 & 100.00 & 98.33 & 70.83 & 96.67 & 81.25 \\
\hline 20 & 98.33 & 87.5 & 100.00 & 91.67 & 100.00 & 100.00 & 100.00 & 75.00 & 99.58 & 88.54 \\
\hline 30 & 100.00 & 91.67 & 100.00 & 95.83 & 100.00 & 100.00 & 100.00 & 79.17 & 100.00 & 91.67 \\
\hline 40 & 100.00 & 91.67 & 100.00 & 95.83 & 100.00 & 100.00 & 100.00 & 7917 & 100.00 & 91.67 \\
\hline 45 & 100.00 & 95.83 & 100.00 & 95.83 & 100.00 & 100.00 & 100.00 & 83.33 & 100.00 & 93.75 \\
\hline 50 & 100.00 & 100.00 & 100.00 & 95.83 & 100.00 & 100.00 & 100.00 & 91.67 & 100.00 & 96.87 \\
\hline 55 & 100.00 & 100.00 & 100.00 & 95.83 & 100.00 & 100.00 & 100.00 & 91.67 & 100.00 & 96.87 \\
\hline
\end{tabular}

Table 2 Accuracy of the classification of pinching $v$. releasing.

\begin{tabular}{|c|c|c|c|c|c|c|c|c|c|c|}
\hline \multicolumn{11}{|c|}{30 Data of Training and 12 Data of Testing } \\
\hline \multirow{3}{*}{$\begin{array}{l}\text { Hidden } \\
\text { neuron }\end{array}$} & \multicolumn{10}{|c|}{ Accuracy } \\
\hline & \multicolumn{2}{|c|}{ Round 1} & \multicolumn{2}{|c|}{ Round 2} & \multicolumn{2}{|c|}{ Round 3} & \multicolumn{2}{|c|}{ Round 4} & \multicolumn{2}{|c|}{ Average } \\
\hline & $\begin{array}{c}\text { Train } \\
(\%)\end{array}$ & $\begin{array}{l}\text { Test } \\
(\%)\end{array}$ & $\begin{array}{c}\text { Train } \\
(\%)\end{array}$ & $\begin{array}{l}\text { Test } \\
(\%) \\
\end{array}$ & $\begin{array}{c}\text { Train } \\
(\%)\end{array}$ & $\begin{array}{l}\text { Test } \\
(\%)\end{array}$ & $\begin{array}{c}\text { Train } \\
(\%)\end{array}$ & $\begin{array}{l}\text { Test } \\
(\%)\end{array}$ & $\begin{array}{c}\text { Train } \\
(\%)\end{array}$ & $\begin{array}{l}\text { Test } \\
(\%)\end{array}$ \\
\hline 1 & 50.00 & 50.00 & 50.00 & 50.00 & 50.00 & 50.00 & 50.00 & 50.00 & 50.00 & 50.00 \\
\hline 5 & 88.33 & 70.83 & 81.67 & 70.83 & 96.67 & 54.17 & 90.00 & 83.30 & 89.17 & 69.78 \\
\hline 10 & 100.00 & 70.83 & 98.33 & 70.83 & 100.00 & 54.17 & 96.67 & 95.83 & 98.75 & 72.92 \\
\hline 20 & 100.00 & 70.83 & 100.00 & 62.50 & 100.00 & 75.00 & 100.00 & 95.83 & 100.00 & 76.04 \\
\hline 30 & 100.00 & 75.00 & 100.00 & 66.67 & 100.00 & 79.17 & 100.00 & 95.83 & 100.00 & 79.17 \\
\hline 40 & 100.00 & 79.17 & 100.00 & 75.00 & 100.00 & 83.33 & 100.00 & 95.83 & 100.00 & 83.33 \\
\hline 45 & 100.00 & 87.50 & 100.00 & 79.17 & 100.00 & 87.50 & 100.00 & 100.00 & 100.00 & 88.54 \\
\hline 50 & 100.00 & 95.83 & 100.00 & 91.67 & 100.00 & 95.83 & 100.00 & 100.00 & 100.00 & 95.83 \\
\hline 55 & 100.00 & 95.83 & 100.00 & 91.67 & 100.00 & 95.83 & 100.00 & 100.00 & 100.00 & 95.83 \\
\hline
\end{tabular}


http://wjst.wu.ac.th

Table 3 Accuracy of the classification of hand lifting $v$. releasing.

\begin{tabular}{|c|c|c|c|c|c|c|c|c|c|c|}
\hline \multicolumn{11}{|c|}{30 Data of Training and 12 Data of Testing } \\
\hline \multirow{3}{*}{$\begin{array}{l}\text { Hidden } \\
\text { neuron }\end{array}$} & \multicolumn{10}{|c|}{ Accuracy } \\
\hline & \multicolumn{2}{|c|}{ Round 1} & \multicolumn{2}{|c|}{ Round 2} & \multicolumn{2}{|c|}{ Round 3} & \multicolumn{2}{|c|}{ Round 4} & \multicolumn{2}{|c|}{ Average } \\
\hline & $\begin{array}{c}\text { Train } \\
(\%)\end{array}$ & $\begin{array}{l}\text { Test } \\
(\%)\end{array}$ & $\begin{array}{c}\text { Train } \\
(\%)\end{array}$ & $\begin{array}{l}\text { Test } \\
(\%)\end{array}$ & $\begin{array}{c}\text { Train } \\
(\%)\end{array}$ & $\begin{array}{l}\text { Test } \\
(\%)\end{array}$ & $\begin{array}{c}\text { Train } \\
(\%)\end{array}$ & $\begin{array}{l}\text { Test } \\
(\%)\end{array}$ & $\begin{array}{c}\text { Train } \\
(\%)\end{array}$ & $\begin{array}{l}\text { Test } \\
(\%)\end{array}$ \\
\hline 1 & 50.00 & 50.00 & 50.00 & 50.00 & 50.00 & 50.00 & 50.00 & 50.00 & 50.00 & 50.00 \\
\hline 5 & 93.33 & 75.00 & 95.00 & 58.33 & 93.33 & 91.67 & 95.00 & 95.83 & 94.16 & 83.33 \\
\hline 10 & 98.33 & 79.17 & 98.33 & 58.33 & 100.00 & 100.00 & 95.00 & 95.83 & 100.00 & 84.38 \\
\hline 20 & 100.00 & 79.17 & 100.00 & 62.50 & 100.00 & 100.00 & 100.00 & 95.83 & 100.00 & 84.38 \\
\hline 30 & 100.00 & 83.33 & 100.00 & 75.00 & 100.00 & 100.00 & 100.00 & 95.83 & 100.00 & 88.54 \\
\hline 40 & 100.00 & 83.33 & 100.00 & 83.33 & 100.00 & 100.00 & 100.00 & 95.83 & 100.00 & 90.62 \\
\hline 45 & 100.00 & 91.67 & 100.00 & 83.33 & 100.00 & 100.00 & 100.00 & 100.00 & 100.00 & 93.75 \\
\hline 50 & 100.00 & 95.83 & 100.00 & 91.67 & 100.00 & 100.00 & 100.00 & 100.00 & 100.00 & 96.86 \\
\hline 55 & 100.00 & 95.83 & 100.00 & 91.67 & 100.00 & 100.00 & 100.00 & 100.00 & 100.00 & 96.86 \\
\hline
\end{tabular}

The $\mathrm{Mu}$ and Beta waves were in the range that correlated with the brain's motoric output [18]. Based on the average accuracies on Tables 1 - 3, Mu and Beta waves calculated using the CSP algorithm as the features showed a good result in helping to separate the 2-class imaginary movement. In a previous study, CSP also demonstrated high accuracies (above $73 \%$ ) for calculating the features (EMG based marker) in distinguishing grasping and release movement combined with the Linear Discriminant Analysis (LDA) as a classifier [19]. The previous study used real movements as features, while this study tried to use imaginary movements to generate the signal like a post-stroke patient who could not move their hand freely. In addition, this study attempted to classified the imaginary movements within the same limb, which was more challenging due to the close spatial representation in the motor cortex area [11].

ELM was proven in giving high accuracies in EEG classification, which were $95 \%$ for the classification of pinching $v$. releasing as the lowest number of accuracy and $96 \%$ for classification of grasping $v$. releasing and lifting hand $v$. releasing as the highest number of accuracy. The lower accuracy showed that pinching was the most laborious task among others. Pinching was challenging to distinguish from the relaxing or releasing movement, moreover in the same upper limb.

The limitations of this study were only focusing on 2-classes of imaginary movement with the releasing movement as a reference in all classification. This study needs more combination of imaginary hand tasks to get more precise performance. For the future work, the 2-class imaginary hand will be combined with a Functional Electrical Stimulator (FES) to help the post-stroke patient in self-exercises during rehabilitation. Hopefully, active rehabilitation which needs the active participation of the patient will benefit recovery or restore hand function more efficiently.

\section{Conclusions}

In this study, the results of a 2-class classification of imaginary (grasping $v$. releasing, pinching $v$. releasing, hand lifting $v$. releasing), analyzed using spectral analysis and CSP calculations was reported. It could be seen in the 3 scatters of distribution data that the feature's values in each class could be better classified after applying the CSP algorithm. Thus, CSP is a useful feature in distinguishing 2 types of hand motion could help Extreme Learning Machine (ELM) to be more precise in classifying the type of 
http://wjst.wu.ac.th

hand movement, even though only a 5-channels electrode EEG was used. The overall average accuracy of the software/system in the classification of 2 types of imaginary hand movement using artificial neural network ELM was above $95 \%$ using 50 hidden neurons for training and testing. This system could be useful for optimizing BCI devices in neuro-rehabilitation, such as combining with a Functional Electrical Stimulator (FES) as a self-therapy for post-stroke patients.

\section{Acknowledgments}

This study was supported by the Faculty of Science and Technology Universitas Airlangga (628/UN3.6/KU/2016). The authors wish to thank all members of the Faculty of Science and Technology who participated in this study.

\section{References}

[1] Badan Penelitian dan Pengembangan Kesehatan Kementrian Kesehatan RI. Riset Kesehatan Dasar. Ministry of Health of the Republic of Indonesia, 2013, p. 129-32.

[2] JA Franck, J Halfens, R Smeets and H Seelen. Concise arm and hand rehabilitation approach in stroke (CARAS): A practical and evidence-based framework for clinical rehabilitation management. Open J. Occup. Ther. 2015; 3, 10.

[3] N Birbaumer, AR Murguialday and L Cohen. Brain-computer interface in paralysis. Curr. Opin. Neurol. 2008; 21, 634-8.

[4] JJ Daly, R Cheng, J Rogers, K Litinas, K Hrovat and M Dohring. Feasibility of a new application of noninvasive brain computer interface $(\mathrm{BCI})$ : A case study of training for recovery of volitional motor control after stroke. J. Neurol. Phys. Ther. 2009; 33. 203-11.

[5] KK Ang, C Guan, KS Phua, C Wang, L Zhou, KY Tang, GJ Ephraim Joseph, CWK Kuah and KSG Chua. Brain-computer interface-based robotic end effector system for wrist and hand rehabilitation: Results of a three-armed randomized controlled trial for chronic stroke. Front. Neuroeng. 2014; 7, 30 .

[6] E Buch, C Weber, LG Cohen, C Braun, MA Dimyan, T Ard, J Mellinger, A Caria, S Soekadar, A Fourkas and N Birbaumer. Think to move: A neuromagnetic brain-computer interface (BCI) system for chronic stroke. Stroke 2008; 39, 910-7.

[7] J Cantillo-Negrete, J Gutierrez-Martinez, RI Carino-Escobar, P Carrillo-Mora and D Elias-Vinas. An approach to improve the performance of subject-independent BCIs-based on motor imagery allocating subjects by gender. Biomed. Eng. Online 2014; 13, 1-15.

[8] R Tomioka, K Aihara and KR Müller. Logistic regression for single trial EEG classification. In: Proceeings of the Neural Information Processing Systems Conference. Canada, 2007, p. 1377-84.

[9] GB Huang, Q Zhu, C Siew, GHÃQ Zhu, C Siew, GB Huang, Q Zhu and C Siew. Extreme learning machine: Theory and applications. Neurocomputing 2006; 70, 489-501.

[10] S Hatamikia and AM Nasrabadi. Subject independent BCI based on LTCCSP method and GA wrapper optimization. In: Proceeings of the $22^{\text {nd }}$ Iranian Conference on Biomedical Engineering. Tehran, Iran, 2015, p. 405-9.

[11] X Yong and C Menon. EEG classification of different imaginary movements within the same limb. PLoS One 2015; 10, 1-24.

[12] M Jochumsen, IK Niazi, K Dremstrup and EN Kamavuako. Detecting and classifying three different hand movement types through electroencephalography recordings for neurorehabilitation. Med. Biol. Eng. Comput. 2016; 54, 1491-501.

[13] P Szachewicz. 2013, Classification of Motor Imagery for Brain-Computer Interfaces, Master Thesis. Poznan University of Technology, Poznan, Poland.

[14] B Shoelson. edfRead. MatlabCentral, 2011.

[15] J Ethridge and W Weaver. Common Spatial Patterns Alogarithm. MatlabCentral, 2009.

[16] Q Yuan, W Zhou, S Li and D Cai. Epileptic EEG classification based on extreme learning machine and nonlinear features. Epilepsy Res. 2011; 96, 29-38. 
http://wjst.wu.ac.th

[17] GB Huang. Introduction to extreme learning machines. In: Hands-on Workshop on Machine Learning for BioMedical Informatics 2006. National University of Singapore, 2006.

[18] MHA Samaha and K AlKamha. Automated classification of L/R hand movement EEG signals using advanced feature extraction and machine learning. Int. J. Adv. Comput. Sci. Appl. 2013; 4, 6.

[19] G Lange, CY Low, K Johar, FA Hanapiah and F Kamaruzaman. Classification of electroencephalogram data from hand grasp and release movements for BCI controlled prosthesis. Proc. Tech. 2016; 26, 374-81.

[20] Emotiv Inc. Emotiv Insight User Manual, Available at: https://www.emotiv.com, accessed November 2017. 